1

5

6 8 Bonington Campus, Loughborough, LE12 5RD, UK.

9

10

11

12

13

14

15

16

17

18

19

20

21

22

23 David Cook ${ }^{\S a}$

11

2
beer

5

§ Corresponding author

\title{
The impact of hop bitter acid and polyphenol profiles on
}

the perceived bitterness of beer.

Olayide Oladokun ${ }^{a}$, Amparo Tarrega ${ }^{a}$, Sue James ${ }^{b}$, Katherine Smart ${ }^{b}$, Joanne Hort ${ }^{a}$,

${ }^{a}$ International Centre for Brewing Science, Bioenergy and Brewing Science Building, University of Nottingham, School of Biosciences, Division of Food Science, Sutton

\section{bSABMiller Plc, SABMiller House, Church Street West, Woking, Surrey, GU21 6HS.}

Abbreviated running title: Impact of bitter congener profiles on perceived bitterness of 
e-mail: david.cook@nottingham.ac.uk

Tel: $+44(0) 1159516245$

26

27 28

Abstract

Thirty-four commercial lager beers were analysed for their hop bitter acid, phenolic acid and polyphenol contents. Based on analytical data, it was evident that the beers had been produced using a range of different raw materials and hopping practices. Principal Components Analysis was used to select a sub-set of 10 beers that contained diverse concentrations of the analysed bitter compounds. These beers were appraised sensorially to determine the impacts of varying hop acid and polyphenolic profiles on perceived bitterness character. Beers high in polyphenol and hop acid contents were perceived as having 'harsh' and 'progressive' bitterness, whilst beers that had evidently been conventionally hopped were 'sharp' and 'instant' in their bitterness. Beers containing light-stable hop products (tetrahydro-iso- $\alpha$-acids) were perceived as 'diminishing', 'rounded' and 'acidic' in bitterness. The hopping strategy adopted by brewers impacts on the nature, temporal profile and intensity of bitterness perception in beer.

Keywords: Beer, phenolic acids, total polyphenol content, hop acids, humulinones, tetrahydro-iso-humulones, bitterness quality.

Chemical compounds studied in this article

Protocatechuic acid (PubChem CID:72); Catechin (PubChem CID:73160); Epicatechin (PubChem CID:72276); Caffeic acid (PubChem CID:689043); Vanillic acid (PubChem CID:8468); Ferulic acid (PubChem CID:445858); $p$-coumaric acid (PubChem CID:637542); Cinnamic acid (PubChem CID:444539); 4-hydroxyphenylacetic acid (PubChem CID:127); Sinapic acid (PubChem CID:637775). 


\section{Introduction}

52

Bitterness is an important flavour character of foods and beverages such as coffee, nuts, fruits and beer (Lesschaeve \& Noble, 2005). Whereas the bitterness flavour of tea and red wine have been attributed mainly to flavonoid phenols, approximately $80 \%$ of beer bitterness is derived from the addition of hops (Humulus lupulus) during the 'boiling stage' of the brewing process (Arrieta, Rodríguez-Méndez, De Saja, Blanco, \& Nimubona, 2010; Caballero, Blanco, \& Porras, 2012). The lupulin glands of female hop cones contain soft resins rich in phloroglucinol derivatives, namely $\alpha$-acids (cohumulone, humulone, adhumulone) and $\beta$-acids (colupulone, lupulone, adlupulone). These acids undergo thermal isomerisation to give iso- $\alpha$-acids, the major bitter compounds in beer (Haseleu et al., 2010). Upon isomerisation, each iso- $\alpha$-acid congener is present as trans/cis stereoisomers with a ratio of approximately $3: 7$ in conventionally hopped beers (Ch Schönberger \& Kostelecky, 2011). In recent years beer-bittering practice has diversified, with the development and usage of hop products in a variety of different forms, and with varied points of addition to the brewing process (e.g. kettle addition, post-fermentation bittering products, or dry hopping, which is feasible at a number of different points). One such product is pre-isomerised iso- $\alpha$-acids, widely available as an aqueous extract or in pellet form, which are prepared from the chemical isomerisation of $\alpha$-acids outside of the brewhouse. These hop products usually have higher levels of cisisomers relative to trans-isomers thus, giving a lower trans/cis ratio (Schmidt et al., 2014). Bitterness can also be achieved by the use of chemically reduced derivatives of iso- $\alpha$-acids, so called light stable hop products such as tetrahydro-iso-humulones (tetra) and hexahydro-iso-humulones (hexa) which are prepared by hydrogenation and reduction reactions, respectively. Advanced hop products are popular among brewers 
because they offer added flexibility in terms of their usage, and can be added downstream of the brewing process (De Keukeleire, 2000).

Furthermore, hops available in various forms (cones, pellets, plugs) can be added at different stages of the brewing process. Some brewers also soak hops in beer during fermentation or conditioning to improve beer aroma in a technique known as 'dryhopping'. Dry-hopping imparts oxidised $\alpha$-acids (known as humulinones) to beer. Humulinones levels of $0.2-0.5 \% \mathrm{w} / \mathrm{w}$ have been reported in hop leaves and pellets (Cocuzza \& Mitter, 2008; Negri, di Santi, \& Tabach, 2010; Wolfe, 2012). In addition to $\alpha$ acids, hops are also a source of polyphenols in beer although the amount of polyphenols present in beer will depend on hop variety, form and the point at which the hops are added during the brewing process. Furthermore, depending on hopping levels, brewing malt usually represents the major source of polyphenols in beer (Aron \& Shellhammer, 2010; Callemien \& Collin, 2009).

Polyphenols contribute to bitterness, colour, body, and astringency in beer and other beverages such as tea and wine, (Collin, Jerkovic, Bröhan, \& Callemien, 2013) and have been recognised to influence the acceptance of beverages (Drewnowski \& GomezCarneros, 2000). In beer they act as antioxidants, preventing oxidative degradation of beer whilst also providing potential health benefits to consumers through their inhibitory activity on certain mutagens and carcinogens (Floridi, Montanari, Marconi, \& Fantozzi, 2003). These compounds are diverse in chemical structure and can be divided into groups consisting of simple hydroxycinnamic and hydroxybenzoic acid derivatives (phenolic acids), flavanols, flavanol glycosides and prenylated flavonoids (Goiris et al., 2014). Flavanols are of particular interest to brewers because they form proteinpolyphenol complexes, leading to the formation of haze or turbidity in beer - brewers consequently remove them by cold filtration or polypvinylpyrrolidine (PVPP) treatment (Garcia, Grande, \& Gándara, 2004). However, PVPP treatment is not selective for the removal of haze active polyphenols only - leading to losses of other polyphenols that are

102 potentially beneficial to the flavour and stability of beer (Aron \& Shellhammer, 2010; 103 Mikyška, Hrabak, Hašková, \& Šrogl, 2002). 
104 The oral sensation of astringency is perceived as a drying, puckering or rough mouth105 feel, resulting from the precipitation of proline-rich proteins in saliva by polyphenols 106 (McLaughlin, Lederer, \& Shellhammer, 2008). Several phenolics including ferulic acid, $p$ 107 coumaric acid and protocatechuic acid have also been noted to elicit astringency 108 (Callemien \& Collin, 2009). Flavanol monomers such as catechin and epicatechin were 109 found to be more bitter than astringent (Drewnowski \& Gomez-Carneros, 2000; Peleg, 110 Gacon, Schlich, \& Noble, 1999).

111 It is widely accepted within the brewing industry that the bitterness characteristics of 112 beers differ due to factors not determined using the simplistic analytical measurement of 113 bitterness units (BU). It is anticipated that this might relate to the diversity of hop 114 products and hopping strategies employed across the industry and the impacts which 115 this has on the relative concentrations of the array of compounds contributing to bitterness perception. Whilst there is some knowledge of the individual bitterness

117 qualities which hop acid isomers impart to beer (Fritsch \& Shellhammer, 2009), the links 118 between hopping practice, bittering congener profile and the perceived bitterness 119 characteristics of beers remains poorly understood. In this study we analysed the major 120 hop acid isomers and polyphenolic compounds present in 34 commercially significant 121 lager beers sourced from around the world. Having thus established the analytical 122 bittering profiles of these beers, 10 beers, which varied significantly in the congeners 123 present, were selected for sensory evaluation. A sensory lexicon for beer bitterness was 124 developed to adequately reflect the diversity of bitterness experienced by the panel and 125 was used to rate beer bitterness characteristics. Finally, correspondence analysis of the 126 sensory data set was used to explore links between the bitterness congener profiles and

127 perceived bitterness character of beers. This study thus represents a significant step 128 towards understanding how to control this important flavour attribute of beers. 


\section{Materials and methods}

$130 \quad 2.1$ Materials

13134 fresh commercial lager beers were sourced from 17 countries over 4 continents and analysed within 8 weeks of production. For reasons of confidentiality the beers are not

133 identified but the countries from which they were sourced are as follows: Australia (2), 134 Belgium (1), Cuba (1), Czech Republic (6), Denmark (1), France (1), Germany (2), 135 Hungary (1), Italy (2), Netherlands (3), Poland (2), Peru (1), Romania (1), South Africa

136 (3), Turkey (1), UK (2) and USA (4).

\subsection{Chemicals and reagents}

Hydroquinone (99\%), catechin (99\%), epicatechin (98\%), 4-hydroxybenzoic acid (99\%), 139 caffeic acid (95\%), vanillic acid (97\%), syringic acid (95\%), p-coumaric acid (98\%), sinapic acid (98\%), ferulic acid (99\%), 2,5-dihydroxybenzoic acid (98\%), gallic acid 141 (98\%), cinnamic acid (98\%), salicylic acid (99\%), 1,2-didydroxybenzene (99\%), 142 homovanillic (99\%), gentisic acid (98\%) and chlorogenic acid (99\%) were all purchased 143 from Sigma-Aldrich (UK). Protocatechuic acid (99.6\%) was acquired from HWI analytic 144 (Germany). Ethyl benzoate, isooctane and methanol (all HPLC grade) as well as 145 orthophosphoric acid 85\% (ASC grade) were purchased from VWR (UK). Reverse osmosis (RO) water was obtained from a Milli-Q water purification system by Millipore.

147 Carboxymethylcellulose (CMC), ethylenediamine tetraacetic acid (EDTA), ammonia and 148 ferric reagent solutions were all technical grade chemicals from VWR (UK). For 149 humulinone synthesis, $\mathrm{CO}_{2}$ extract of $\alpha$-acid resin (86\%) was kindly donated by Botanix, 150 Paddock Wood, Kent. Cumene hydroperoxide ( $80 \%$ technical grade), diethyl ether, sodium bicarbonate, hexane, phosphoric acid and hydrochloric acid $(\mathrm{HCl})$ were all from

152 Sigma-Aldrich (UK) and of ASC reagent grades.

Iso-a-acid standard (ICE-3) containing trans-isocohumulone, trans-isohumulone, transisoadhumulone $(62.3 \% \mathrm{w} / \mathrm{w}), \alpha-\& \beta$-acid $(44.64 \%, 24.28 \% \mathrm{w} / \mathrm{w})$, and tetra standard

155 (99.3\% w/w) were purchased from Labor Veritas Co. (Switzerland). 


\subsection{Instrumentation}

157 HPLC analysis was carried out on a Waters Alliance 2695 instrument equipped with a 158 column heater and a membrane degasser. Detection was achieved with a UV detector 159 and peak areas were processed with the operating HPLC software (Empower 2). 160 Separation of polyphenols and hop acids was achieved with a Purospher STAR rp-18 161 endcapped column ( $250 \times 4.6 \mathrm{~mm}, 3 \mu \mathrm{m})$ from Merck Millipore (UK) coupled with a C18 guard cartridge from Phenomenex (UK).

\subsection{Analysis of hop bitter acids in beer}

\subsubsection{Extraction of hop bitter acids from beer}

165 Cold beer was degassed by stirring for $1 \mathrm{~h}$ followed by the transfer of an aliquot ( $5 \mathrm{ml}$ ) 166 into a $50 \mathrm{ml}$ centrifuge tube, the degassed beer was acidified with orthophosphoric acid $167(100 \mu \mathrm{l})$ and an internal standard (benzoic acid) was added $(0.003 \mathrm{mg} / \mathrm{L})$. The mixture 168 was then extracted into isooctane $(10 \mathrm{ml})$ on a roller bed for $30 \mathrm{~min}$. The isooctane 169 extract was transferred into a glass tube and evaporated under a controlled flow of 170 Nitrogen with a Visidry attachment coupled to a solid phase extraction manifold 171 (Supelco). The residue was dissolved in acetonitrile $(2 \mathrm{ml})$ to give the HPLC sample.

\subsubsection{HPLC-UV analysis of hop bitter acids}

173 Hop acid separation was achieved with a binary mixture of (A) $1 \% \mathrm{v} / \mathrm{v}$ acetic acid and 174 (B) $0.1 \% \mathrm{v} / \mathrm{v}$ orthophosphoric acid in acetonitrile. The gradient elution was: 0-5 min: $17530 \% A, 70 \%$ B; $15-24 \min : 20 \% A, 80 \%$ B; $25 \min : 10 \% A, 90 \% B ; 30 \min : 10 \% A$, $17690 \%$ B; $35 \min : 0 \%$ A, 100\% B; $44 \min : 0 \%$ A, 100\% B; $46 \min : 30 \%$ A, 70\% B; 55 $177 \min : 30 \% A, 70 \%$ B over a 55 min run time. Injection volume was $10 \mu l$, flow rate was $1780.5 \mathrm{ml} / \mathrm{min}$ and column temperature was $25^{\circ} \mathrm{C}$. The peak area of iso- $\alpha$-acids, 179 humulinones were extracted at $270 \mathrm{~nm}$ and at $310 \mathrm{~nm}$ for tetrahydro-iso- $\alpha$-acids.

\subsubsection{Determination of bitterness units}



Analysis, 2011). Beer $(5 \mathrm{ml})$ was transferred into a $50 \mathrm{ml}$ centrifuge tube and acidified with $3 \mathrm{~N} \mathrm{HCl}(0.5 \mathrm{ml})$. Isooctane $(10 \mathrm{ml})$ was added and the mixture was shaken by hand three times before extraction on a rolled bed for $15 \mathrm{~min}$. The mixture was subsequently centrifuged at $400 \times \mathrm{g}$ twice for $5 \mathrm{~min}$ each time to aid phase separation. An aliquot of the clear isooctane layer was transferred into a cuvette and absorbance was measured with a spectrophotometer at $275 \mathrm{~nm}$ against a blank of orthophosphoric acid and isooctane. The recorded absorbance was multiplied by an empirical factor of 50 to give $\mathrm{BU}$ values in $\mathrm{mg} / \mathrm{L}$.

\subsection{Analysis of phenolic/ polyphenol compounds in beer}

\subsubsection{Extraction of beer phenolic compounds}

The phenolic compounds listed in section 2.2 were extracted from beer using liquid-liquid extraction. Degassed beer $(5 \mathrm{ml})$ was transferred into a $50 \mathrm{ml}$ centrifuge tube before acidification with orthophosphoric acid $(250 \mu \mathrm{l})$. Ethyl acetate $(10 \mathrm{ml})$ was then added before extraction on a roller bed for $30 \mathrm{~min}$. After extraction, the residual beer from the bilayer mixture was discarded and RO water $(5 \mathrm{ml})$ was added and further extracted on the roller bed for $15 \mathrm{~min}$. The water layer was removed and discarded while the extract in ethyl acetate was transferred into a glass tube and dried down under controlled flow of Nitrogen using a Visidry attachment coupled to a SPE manifold (Supelco). The residue was reconstituted in a fixed volume of methanol $(2 \mathrm{ml})$ prior to HPLC analysis.

\subsubsection{HPLC-UV analysis of beer phenolic compounds}

202 The chromatographic method used a binary solvent system consisting of (A) $1.25 \% \mathrm{v} / \mathrm{v}$ acetic acid and (B) $0.1 \% \mathrm{v} / \mathrm{v}$ orthophosphoric acid in acetonitrile. The gradient elution protocol was as follows: $0-25 \min : 98 \% A, 2 \% B ; 25-30 \min : 76 \%$ A, 24\% B; $35-40$ $\min : 55 \%$ A, 45\% B; $45 \min : 15 \%$ A, 85\% B; $50 \min : 0 \% A, 100 \%$ B; $55-65 \min : 98 \%$ A, $2 \%$ B. Injection volume was $10 \mu \mathrm{l}$, flow rate was $0.5 \mathrm{ml} / \mathrm{min}$ and column temperature 
Beer total polyphenol content (TPC) was determined according to ASBC method Beer-35 (ASBC Method of Analysis, 1978), involving the reaction of polyphenols with ferric ion in an alkaline solution. Beer $(10 \mathrm{ml})$ was mixed with a preparation of

212 carboxymethylcellulose (CMC, 1\%) and ethylenediamine tetraacetic acid (EDTA, 0.2\%) $213(8 \mathrm{ml})$ in a $25 \mathrm{ml}$ volumetric flask. Ferric acid $(0.5 \mathrm{ml})$ was added, followed by ammonia $214(0.5 \mathrm{ml})$ with mixing after each addition. The solution was then made up to mark with 215 RO water and left to stand at room temperature for $10 \mathrm{~min}$ before an absorption 216 measurement was taken at $600 \mathrm{~nm}$. The recorded absorbance was multiplied by 820 to give total polyphenol values in $\mathrm{mg} / \mathrm{L}$.

\subsection{Synthesis of humulinones from humulones}

Humulinones were synthesised from humulone resin prepared from $\mathrm{CO}_{2}$ extract of hops (86.3\% $\alpha$-acids) using a modified version of a reported method (Taniguchi, Matsukura, Ozaki, Nishimura, \& Shindo, 2013). Humulone $(1.41 \mathrm{~g})$ and cumene hydroperoxide $(0.7$ $\mathrm{ml}$ ) were dissolved in diethyl ether $(7 \mathrm{ml})$. A solution of saturated sodium bicarbonate $\left(\mathrm{NaHCO}_{3}, 6 \mathrm{~g}\right.$ dissolved slowly in $40 \mathrm{ml} \mathrm{RO}$ water) was added to the solution and kept at room temperature in a sealed vessel for 5 days, after which the sodium salt of humulinones was generated. The salt was filtered and washed with water $(150 \mathrm{ml} \times 2)$ and diethyl ether $(150 \mathrm{ml} \times 2$ ) under vacuum in a Buchner flask and funnel. The crude extract $(1.45 \mathrm{~g})$ was subsequently dissolved in methanol $(100 \mathrm{ml})$ containing $1 \% \mathrm{v} / \mathrm{v}$ phosphoric acid before the addition of a $0.5 \mathrm{~N} \mathrm{HCl}$ solution $(800 \mathrm{ml})$. The mixture was partitioned with hexane $(1 \mathrm{~L} \times 2)$ before the hexane layer was evaporated to dryness with a rotary evaporator to yield humulinones $(0.95 \mathrm{~g})$ of $99 \%$ purity (by HPLC).

\subsection{Sensory evaluation of bitterness}

232 Ethical approval for the sensory element of this investigation was obtained from the 233 University of Nottingham Medical Ethics Committee (J12022015) and all participants gave written informed consent to participate in the study. 
235 The qualitative aspects of bitterness were evaluated by experienced panellists from the 236 University of Nottingham trained beer panel $(n=6)$ using descriptive analysis. First, 237 panellists were presented with a subset of 10 of the 34 beers to generate and define a 238 bitterness lexicon. These beers represented extreme variation in analytical variables and were selected based on a PCA plot from the analytical concentration of their hop acid and polyphenol contents. Panellists then attended a further $22 \mathrm{~h}$ sessions during which they tasted and described the bitterness of $10 \mathrm{ml}$ samples of each beer and participated in 242 group discussions to agree a final list of clearly defined bitterness related terms. Beer samples $(10 \mathrm{ml})$ were then evaluated in 2 further sessions using a Check-All-That-Apply (CATA) technique, (Dooley, Lee, \& Meullenet, 2010) where panellists were asked to indicate which of the terms in the lexicon were relevant to each sample. Data was collected with Fizz software (Biosystèmes, France). Each sample was presented individually and assessed in triplicate following a randomised balanced order based on a partial latin square design and served at $4 \pm 1^{\circ} \mathrm{C}$. During sample evaluation, panellists were given 5 min to evaluate each sample followed by a 3 min break in order to minimise bitterness carry over. Water (Evian, Danone, France) and crackers (Rakusen's, UK) were provided for palate cleansing.

\subsection{Quantitation and statistical analysis}

External standard solutions of a-acids $(0.5,1,2,4$ and $8 \mathrm{mg} / \mathrm{L})$, iso- $\alpha$-acids $(1,10,20$, 40 and $60 \mathrm{mg} / \mathrm{L})$, tetra $(0.5,1,2,4$ and $8 \mathrm{mg} / \mathrm{L})$ and humulinones $(0.5,1,2,4$ and 8 $\mathrm{mg} / \mathrm{L}$ ) were all prepared in acetonitrile. The mean values of triplicate injections were used to plot calibration curves for the quantification of hop bitter acids in beers. A stock solution $(20 \mathrm{mg} / \mathrm{L})$ of standard grade phenolic compounds was prepared by dissolving $(0.01 \mathrm{~g})$ of the standards in a $500 \mathrm{ml}$ volumetric flask containing a mixture of RO water and methanol ( $200 \mathrm{ml}$ ), before making up to mark with the same solvent mixture. Serial dilution was made from the stock solution to achieve external standards of 10,5 , 2.5, 1 and $0.5 \mathrm{mg} / \mathrm{L}$ levels. Quantification was achieved from the standard calibration curves. Statistical analysis including Cochran's $\mathrm{Q}$ test was used to determine which of 
263 the attributes were significantly different between the beers. Correspondence analysis 264 was used to process the frequency data of bitterness attributes for each beers. Principal 265 component analysis (PCA) was used to aid the selection of beers samples for sensory 266 analysis based on analytical measurements of hop acid and polyphenol contents. All 267 statistical analyses were performed with the XLSTAT, v2015 package. 


\section{Results and discussion}

\subsection{Phenolic profiles of lager beers}

270 The liquid-liquid extraction protocol using ethyl acetate and water enabled the effective analysis of quantitatively significant phenolic compounds in beer. An example of the

272 chromatographic separation achieved with the described extraction protocol and HPLC method for the Czech lager beer $(L)$ is provided as complementary data. The HPLC method described enabled the simultaneous separation and quantification of several phenolics in beer. Where possible, the phenolic compounds were identified based both on prior knowledge and by matching peaks against authentic standards run separately, and with regard to both retention time and UV absorbance spectrum. Whilst not all peaks on the trace could be identified, unknown peak areas were also integrated and included in the analytical profiles of the beers labelled as unknown (U) $1,2 \ldots$. etc. The elution pattern of phenolic acids in beer followed an order of decreasing polarity under RP-HPLC conditions, thus phenolic acid derivatives of benzoic acid were eluted before the hydroxycinnamic acid derivatives. The polarity of phenolic acids is increased mostly by the hydroxyl group at the para-position, followed by the ortho- and meta-positions of the benzene ring (Torres, Mau-Lastovicka, \& Rezaaiyan, 1987). The phenolic profile of each of the 34 beers was analysed, however, without further reference to beer brands it would not be informative to publish this data for each 'blind-coded' beer. To illustrate the variability present in the data set, we summed the total contents of the quantified phenolic compounds in each beer (Table 1), which shows a substantial range of concentrations ( 3.9 to $21.2 \mathrm{mg} / \mathrm{L}$ ). Ferulic acid was the most abundant phenolic acid present in the beers, with a concentration ranging from $0.98 \mathrm{mg} / \mathrm{L}$ in the Australian lager (BB) to $7.61 \mathrm{mg} / \mathrm{L}$ in the American lager beer $\mathrm{O}$ (data not shown). $p$-coumaric acid is the precursor compound to ferulic acid and is formed via the shikimic acid reaction pathway, therefore the concentration of ferulic acid is usually greater than that of $p$-coumaric acid in beer (Garcia et al., 2004). The concentrations of $p$-coumaric acid across the beers followed a similar pattern as observed for ferulic acid, with beers BB and O containing 

contain the highest amount of phenolic compounds overall (Table $1 ; 21.17 \mathrm{mg} / \mathrm{L}$ ), while beer $\mathrm{F}$ a South African lager beer had the lowest concentration at $3.91 \mathrm{mg} / \mathrm{L}$. Beers brewed in Germany (J, P), South Africa (F, C, D), Denmark (S) and Australia (BB, DD) all had phenolic compound concentrations below $8 \mathrm{mg} / \mathrm{L}$ whilst the Czech beers (I, E, L, $\mathrm{HH}$ ) and American lagers $(\mathrm{O}, \mathrm{T}, \mathrm{Q})$ all had phenolic compound concentrations of $>10$ $\mathrm{mg} / \mathrm{L}$. These values represent the total free phenolic acid content of beer which is reported to be approximately $10-20 \%$ of total beer polyphenol content, since a significant portion of beer phenolics are suggested to exist in bound form (Floridi et al., 2003). The Czech beer $(\mathrm{HH})$ was the only sample that contained gallic acid whilst catechol, chlorogenic, salicylic, homovanillic and gentisic acids were not detected in the beers, in agreement with reports from other studies (Garcia et al., 2004; Jandera et al., 308 2005).

3.2 The relationship between phenolic acid and total polyphenol content of beer

The TPC of the beers was found to range between 74 and $256 \mathrm{mg} / \mathrm{L}$. Similar values (70 $240 \mathrm{mg} / \mathrm{L}$ ) were reported by Dvorakova et al. (2007). The lowest concentration was found in the Hungarian lager beer $(\mathrm{G})$ whilst beers $\mathrm{O}$ and $\mathrm{T}$, both American lagers and the British lager beer (AA) all had polyphenol contents greater than $250 \mathrm{mg} / \mathrm{L}$. Beer $\mathrm{O}$ contained the highest amount of phenolic compounds and total polyphenol content. A plot of total phenolic compound concentration versus TPC is displayed in Fig. 1. The plot has been annotated to show three main clusters. The first cluster is of beers characterised by TPC values of approximately $74-180 \mathrm{mg} / \mathrm{L}$ and phenolic compound contents ranging between 3 and $15 \mathrm{mg} / \mathrm{L}$. Beers $\mathrm{AA}$ and $\mathrm{T}$ formed a separate cluster, due to the high TPC in these beers (>250 mg/L). In the last cluster, consisting of beers $\mathrm{E}, \mathrm{O}$ and $\mathrm{HH}$, TPC ranged from $145-253 \mathrm{mg} / \mathrm{L}$ and phenolic compounds were in excess of $15 \mathrm{mg} / \mathrm{L}$. Interestingly, this data shows that there was no linear relationship between

322 TPC and the sum of phenolic compounds quantified. This is most likely due to the highly varied brewing techniques and ingredients employed in the industry. The observed 
324 higher TPC concentrations ( $>250 \mathrm{mg} / \mathrm{L}$ ) in beers $\mathrm{T}, \mathrm{AA}$ and $\mathrm{O}$ indicates that these beers

325 were dry-hopped products.

326

327

328

329

330

331

332

333

\subsection{Hop bitter acid profile of lager beers}

For the bitterness profiles of these beers, the bitter tasting hop acids present in the lager beers were evaluated using two separate analytical methods; firstly by HPLC as described in section 2.4 .2 and secondly by bitterness unit method (2.4.3). The latter method has been suggested to yield inflated bitterness values due its susceptibility to interference from other compounds present in beer that absorb light at the wavelength of measurement (Schönberger, 2006; Tomlinson, Ormrod, \& Sharpe, 1995). In contrast, HPLC measurements are agreed to provide a better assessment of beer bitterness because they allow for the selective quantification of iso- $\alpha$-acids, the major bittering principles in beer (Ting, Kay, \& Ryder, 2007). A comparison of the hop bitter acid concentrations in the beers by BU and HPLC methods is presented in Fig. 2 . The results showed that the BU of the lager beers ranged from $8-36 \mathrm{mg} / \mathrm{L}$, although bitterness was overestimated by the BU method in comparison to HPLC values in around $60 \%$ of the samples. The sum of analysed iso- $\alpha$-acid hop acid concentrations (HPLC) was between 8 and $41 \mathrm{mg} / \mathrm{L}$. The average bitterness across all 34 beers in both methods was $23 \mathrm{mg} / \mathrm{L}$ and the most bitter beers had concentrations $>30 \mathrm{mg} / \mathrm{L}$ ( $G \mathrm{G}, A A$ and $\mathrm{L}$ ). The latter beers each had lower BU values in comparison to HPLC values, e.g. beer AA had a BU value of $34 \mathrm{mg} / \mathrm{L}$ but the HPLC value was $41 \mathrm{mg} / \mathrm{L}$. Around 7 of the beers including U, J, I, E, C and $\mathrm{T}$ had similar bitterness concentrations according to both methods. The American light lager beer $\mathrm{R}$ contained the lowest amount of hop acids ( $8 \mathrm{mg} / \mathrm{L})$ and did not contain any reduced iso- $\alpha$-acid products.

Inspection of the HPLC chromatograms revealed that both iso- $\alpha$-acids and tetrahydroiso- $\alpha$-acids were present in beers $\mathrm{K}, \mathrm{S}, \mathrm{V}, \mathrm{N}$, as well as the presence of humulinones at up to $3 \mathrm{mg} / \mathrm{L}$ in beers $\mathrm{O}, \mathrm{Q}, \mathrm{AA}, \mathrm{T}$ and $\mathrm{V}$. The presence of tetrahydro-iso- $\alpha$-acids in beers $\mathrm{K}, \mathrm{S}, \mathrm{V}, \mathrm{N}$ explains the lower $\mathrm{BU}$ values attained in these beers relative to the values 
determined by HPLC, since BU absorbance is taken at a lower wavelength (275 nm) to the absorption maxima of tetrahydro-iso- $\alpha$-acids $(310 \mathrm{~nm})$. Significantly, of these four beers the $\mathrm{BU}$ value of beer $\mathrm{V}$ which contained humulinones was the only one greater than the attained HPLC value. This was also the case in the other beers containing humulinones ( $\mathrm{O}, \mathrm{Q}$ and $\mathrm{T}$ ) except for beer AA. This perhaps suggests that humulinones contribute to the bitterness values attained with BU method as has been previously observed (Parkin, 2014), although the contribution of beer polyphenols to BU values cannot be totally excluded either. The presence of humulinones as well as relatively high polyphenol content in beers $\mathrm{O}, \mathrm{Q}, \mathrm{AA}, \mathrm{T}$ and $\mathrm{V}$ further supports the hypothesis that these beers were dry-hopped. The low TPC observed in beer $Q$ in comparison to the other dryhopped beers could be explained by a lower rate of dry-hopping or dry-hopping with different hop products, i.e. hop pellets which contain relatively lower polyphenol content instead of whole hop cones. Beers that contained tetra hop products $(K, S, N, V)$ and those dry-hopped ( $Q, A A, T, O)$ both displayed distinctive polyphenol and bitter acid profiles. The highly dry-hopped beers $(T, A A, Q)$ and high bitterness Czech lagers ( $E$ and L) all had correspondingly high contents of phenolic compounds (see Table 1 and Figure 2).

\subsection{Selection of exemplar beers for sensory assessment}

In order to understand how the varying contents of hop acid isomers and phenolic compounds impact on perceived sensory bitterness, a sub-set of 'exemplar beers' were selected with the aid of a PCA plot of the analytical data. The PCA bi-plot shown in Fig. 3 accounted for about $65 \%$ of variation within the data set. A negative loading on PC 1

$373(47.6 \%$ of variation) was associated with the use of tetrahydro-iso- $\alpha$-acids, whilst 374 positive loadings on this axis were related to high levels of iso- $\alpha$-acids, $\alpha$-acids, humulinones and phenolic compounds. A positive loading on PC2 identified beers with a high trans/cis ratio and residual $\alpha$-acids - i.e. those which had used conventional

377 hopping practice as opposed to pre-isomerised or light stable products. Negative 
378 loadings on this axis were driven largely by phenolic compounds (quadrant 4) or

379 tetrahydro-iso- $\alpha$-acids (quadrant 3 ).

380 Beers in quadrant 1 were generally lower in hop acid and polyphenol content compared

381 to beers in quadrant 4 which were characterised by high levels of these compounds. The 382 beers in quadrant 2 were correlated with high trans/cis ratio and residual $\alpha$-acids which, 383 as noted, is indicative of conventional hopping techniques. Beers in quadrant 3 had lower 384 trans/cis ratios, (indicative of the use of pre-isomerised hops) as well as containing 385 tetrahydro-iso- $\alpha$-acids. A total of 10 beers were selected from the 4 quadrants to 386 represent the diversity amongst the 34 beers: beers $C C$ and $V$ from quadrant $1, X$ and 387 GG from quadrant $2, \mathrm{~S}, \mathrm{~N}$ and $\mathrm{BB}$ from quadrant 3 and beers $\mathrm{E}, \mathrm{AA}$ and $\mathrm{T}$ from quadrant 3884.

\subsection{Beer bitterness lexicon}

390 A total of 13 bitterness descriptors were generated by the trained panel of beer tasters 391 following concept alignment. These attributes as well as their definitions are presented in 392 Table 2, with some of the attributes e.g. instant, diminishing and progressive notably 393 related to the temporal character of bitterness. Cochran's Q test analysis of the CATA 394 frequency data showed that only 4 of the 13 bitterness attributes (acidic, tart, astringent 395 and artificial) did not significantly differentiate across the sample set ( $p>0.05)$ (Table 2). The temporal descriptors as well as descriptors such as harsh, rounded, metallic and smooth were all rated significantly differently amongst the 10 beers $(p<0.05)$. The correspondence analysis of the sensory data is presented in Fig. 4. This revealed that beer CC, selected from quadrant 1 of the PCA in figure 3, which had relatively low hop bitter acid and polyphenol contents, was perceived as having an 'artificial', 'metallic' and 'instant' bitterness. Beer $\mathrm{V}$ from the same quadrant (figure 3), but deduced to have been dry-hopped from the presence of humulinones, had a 'rounded' and 'smooth' bitterness character; temporally this beer was 'diminishing' in bitterness. Conventionally 
bittered beer GG with a high trans/cis ratio (selected from quadrant 2 of the PCA) was perceived as having a 'sharp' and 'instant' bitterness. Beers $\mathrm{N}$ and $\mathrm{S}$ from quadrant 3 of the PCA which were bittered with a blend of tetra as well as iso- $\alpha$-acids were described as 'diminishing' and somewhat 'acidic' in bitterness. Considering the trained panel was not aware of the analytical bitterness fingerprint of these beers, it is interesting that all the beers containing tetra $(S, N$, and $V)$ are grouped together based on their sensory bitterness character in the upper right quadrant of Fig. 4. Furthermore, they were negatively correlated in this plot with beers $A A, T$ and $E$ which were relatively high in levels of humulinones, iso- $\alpha$-acids and polyphenols. This further supports the notion that besides the intensity of bitterness, the character of bitterness in beer is also impacted by the type of hop product used for bittering. The contribution of humulinones to beer bitterness character is not yet fully understood. They were first thought not to contribute significantly to bitterness (Verzele, 1986) but recent publications have associated the presence of humulinones in beer with an increased sensation of bitterness and potentially the source of harsh bitterness character often present in dry-hopped beers (McLaughlin et al., 2008; Parkin, 2014). A recent report by Hopsteiner suggested that humulinones are approximately $65 \%$ as bitter as iso- $\alpha$-acids, thus representing a significant additional source of bitterness in beer (Steiner, 2015).

The third beer selected from quadrant 3 (BB) which was exclusively bittered with pre424 isomerised products (based on the analytical profile and lower trans/cis ratio) was 425 described as being 'vegetative' in bitterness. Beers AA, E and T from quadrant 4 of the 426 PCA, containing the highest concentrations of hop acids, humulinones and polyphenols were described as having a 'harsh' and 'progressive' bitterness character. A study of the 428 interaction between iso- $\alpha$-acids and hop polyphenols by time-intensity (TI) and free 429 choice profiling (FCP) as reported by McLaughlin et al. (2008) found a significant effect of polyphenols on perceived intensity, as well as character, of bitterness. In the study, samples high in polyphenols were higher in intensities of 'harsh', 'medicinal', and 
432 'metallic' (McLaughlin et al., 2008). Our results for the bitterness characters of beers AA, $433 \quad \mathrm{E}$ and $\mathrm{T}$ in particular are in agreement with those findings. 


\section{Conclusions}

435 The phenolic acid and total polyphenol contents of 34 lager beers brewed in different 436 geographical locations were determined. The former was achieved by a combination of 437 LLE and HPLC analysis while the latter was accomplished with an international global assay method. Phenolic compound concentrations ranged from $3-12 \mathrm{mg} / \mathrm{L}$ and TPC was between $74-256 \mathrm{mg} / \mathrm{L}$, with the highest values identified in dry-hopped beers. No linear relationship was found between total phenolic compound concentration and TPC although dry-hopped beers were found to contain a greater amount of polyphenols in comparison

442 to beers that had not been dry-hopped (N.B. the usage of dry hopping was deduced 443 from the presence of significant concentrations of humulinones in the beer, but was not verified by the manufacturers in all instances). Sensory analysis showed that beers with varying profiles of bitter congeners (hop acids and phenolics) had distinctive bitterness characters. These differences are believed to be driven by the selective usage of various hop products and points of addition in the brewing process. Since the present work used a survey of international lager brands, these factors are largely deduced, albeit logically and based on obvious analytical differences between the finished beers; however, it should be borne in mind that the manufacturing processes were not disclosed, nor were they independent variables in the study. Dry-hopped beers generally contained more polyphenol compounds and humulinones, and were sensorially perceived as having a 'harsh' and 'progressive' bitterness. In comparison, beers which had evidently been conventionally bittered and as such contained relatively high residual amount of $\alpha$-acids and trans/cis ratio were perceived as having an 'instant' and 'sharp' bitterness. Beers containing tetrahydro-iso- $\alpha$-acids were rated as having a 'diminishing' temporal character of bitterness. These results support the hypothesis that the production processes employed by brewers in terms of hopping strategy, and the raw materials used, give beers a distinct polyphenolic and bitterness fingerprint which influences the overall bitterness impression of beer. Understanding the sensory character of bitterness in beers, and how that relates to their analytical bitterness fingerprint is of significant 
462 value in order to both understand consumer response to beer bitterness and to optimise 463 production processes in this regard.

\section{Acknowledgement:}

465 We gratefully acknowledge the financial support of SABMiller plc and the University of 466 Nottingham in sponsoring this research. 
468

469

470

471

472

473

474

475

476

477

478

479

480

481

482

483

484

485

486

487

488

489

490

491

492

493

494

495

496

497

498

499

500

501

502

503

504

505

506

507

Aron, Patricia M, \& Shellhammer, Thomas H. (2010). A discussion of polyphenols in beer physical and flavour stability. Journal of the Institute of Brewing, 116(4), 369-380.

Arrieta, Álvaro A, Rodríguez-Méndez, María L, De Saja, Jose A, Blanco, Carlos A, \& Nimubona, Dieudonné. (2010). Prediction of bitterness and alcoholic strength in beer using an electronic tongue. Food Chemistry, 123(3), 642-646.

ASBC Method of Analysis. (1978). American Society of Brewing Chemists. Total Polyphenol, Beer-35. The Society: St Paul, MN, U.S.A.

ASBC Method of Analysis. (2011). American Society of Brewing Chemists. Beer Bitterness, Beer-23A. The Society: St Paul, MN, U.S.A.

Caballero, Isabel, Blanco, Carlos A, \& Porras, María. (2012). Iso- $\alpha$-acids, bitterness and loss of beer quality during storage. Trends in Food Science \& Technology, 26(1), 21-30.

Callemien, Delphine, \& Collin, Sonia. (2009). Structure, organoleptic properties, quantification methods, and stability of phenolic compounds in beer - A review. Food Reviews International, 26(1), 1-84.

Cocuzza, Sandro, \& Mitter, Willi. (Personal Communication). Dry Hopping - A Study Of Various Parameters. Hopsteiner Technical Publications.

Collin, Sonia, Jerkovic, Vesna, Bröhan, M, \& Callemien, D. (2013). Polyphenols and Beer Quality Natural Products: Springer. (pp. 2333-2359).

De Keukeleire, Denis. (2000). Fundamentals of beer and hop chemistry. Quimica nova, 23(1), 108-112.

Dooley, Lauren, Lee, Young-seung, \& Meullenet, Jean-François. (2010). The application of check-all-that-apply (CATA) consumer profiling to preference mapping of vanilla ice cream and its comparison to classical external preference mapping. Food Quality and Preference, 21(4), 394-401.

Drewnowski, Adam, \& Gomez-Carneros, Carmen. (2000). Bitter taste, phytonutrients, and the consumer: a review. The American Journal of Clinical Nutrition, 72(6), 14241435.

Floridi, Simona, Montanari, Luigi, Marconi, Ombretta, \& Fantozzi, Paolo. (2003). Determination of free phenolic acids in wort and beer by coulometric array detection. Journal of Agricultural and Food Chemistry, 51(6), 1548-1554.

Fritsch, Annette, \& Shellhammer, Thomas H. (2009). The Bitter Qualities of Reduced and Nonreduced Iso- $\alpha$-acids. Journal of the American Society of Brewing Chemists, 67(1), 8-13.

Garcia, A Alonso, Grande, B Cancho, \& Gándara, J Simal. (2004). Development of a rapid method based on solid-phase extraction and liquid chromatography with ultraviolet absorbance detection for the determination of polyphenols in alcohol-free beers. Journal of Chromatography A, 1054(1), 175-180.

Goiris, Koen, Jaskula-Goiris, Barbara, Syryn, Evelien, Van Opstaele, Filip, De Rouck, Gert, Aerts, Guido, \& De Cooman, Luc. (2014). The flavoring potential of hop polyphenols in beer. Journal of the American Society of Brewing Chemists, 72(2), 135-142. 
Haseleu, Gesa, Lagemann, Annika, Stephan, Andreas, Intelmann, Daniel, Dunkel, Andreas, \& Hofmann, Thomas. (2010). Quantitative sensomics profiling of hop-derived bitter compounds throughout a full-scale beer manufacturing process. Journal of Agricultural and Food Chemistry, 58(13), 7930-7939.

Jandera, Pavel, Škeříková, Veronika, Řehová, Lucie, Hájek, Tomáš, Baldrianová, Lucie, Škopová, Gabriela, . . . Horna, Aleš. (2005). RP-HPLC analysis of phenolic compounds and flavonoids in beverages and plant extracts using a CoulArray detector. Journal of Separation Science, 28(9-10), 1005-1022.

Lesschaeve, Isabelle, \& Noble, Ann C. (2005). Polyphenols: factors influencing their sensory properties and their effects on food and beverage preferences. The American Journal of Clinical Nutrition, 81(1), 330S-335S.

McLaughlin, Ian R, Lederer, Cindy, \& Shellhammer, Thomas H. (2008). Bitternessmodifying properties of hop polyphenols extracted from spent hop material. Journal of the American Society of Brewing Chemists, 66(3), 174-183.

Mikyška, A, Hrabak, M, Hašková, D, \& Šrogl, J. (2002). The role of malt and hop polyphenols in beer quality, flavour and haze stability. Journal of the Institute of Brewing, 108(1), 78-85.

Negri, Giuseppina, di Santi, Daniel, \& Tabach, Ricardo. (2010). Bitter acids from hydroethanolic extracts of Humulus lupulus L., Cannabaceae, used as anxiolytic. Revista Brasileira de Farmacognosia, 20(6), 850-859.

Parkin, Ellen Jane. (Unpublished results). The influence of polyphenols and humulinones on bitterness in dry-hopped beer.

Peleg, Hanna, Gacon, Karine, Schlich, Pascal, \& Noble, Ann C. (1999). Bitterness and astringency of flavan-3-ol monomers, dimers and trimers. Journal of the Science of Food and Agriculture, 79(8), 1123-1128.

Schmidt, Christina, Biendl, Martin, Lagemann, Annika, Stettner, Georg, Vogt, Christian, Dunkel, Andreas, \& Hofmann, Thomas. (2014). Influence of Different Hop Products on the cis/trans Ratio of Iso- $\alpha$-Acids in Beer and Changes in Key Aroma and Bitter Taste Molecules During Beer Ageing. Journal of the American Society of Brewing Chemists, 72(2), 116-125.

Schönberger, Ch, \& Kostelecky, T. (2011). 125th Anniversary Review: the role of hops in brewing. Journal of the Institute of Brewing, 117(3), 259-267.

Schönberger, Christina. (2006). Bitter is better. Monatsschrift für Brauwissenschaft, 3(4), 5665.

Steiner, S. (Personal Communication). Humulinone Utilization In Dry-Hopped Beers. Hopsteiner Newsletter.

Taniguchi, Yoshimasa, Matsukura, Yasuko, Ozaki, Hiromi, Nishimura, Koichi, \& Shindo, Kazutoshi. (2013). Identification and quantification of the oxidation products derived from $\alpha$-acids and $\beta$-acids during storage of hops (Humulus lupulus L.). Journal of Agricultural and Food Chemistry, 61(12), 3121-3130.

Ting, Patrick L, Kay, Susan, \& Ryder, David. (2007). Separation and identification of stereoisomers of isomerized $\alpha$-acid derivatives by HPLC/DAD and electrospray HPLC/MS. Journal of the American Society of Brewing Chemists, 65(1), 9-14. 
551 Tomlinson, JB, Ormrod, IHL, \& Sharpe, FR. (1995). A novel method for bitterness determination in beer using a delayed fluorescence technique. Journal of the Institute of Brewing, 101(2), 113-118.

554 Torres, Andrew M, Mau-Lastovicka, Terry, \& Rezaaiyan, Ruhi. (1987). Total phenolics and high-performance liquid chromatography of phenolic acids of avocado. Journal of Agricultural and Food Chemistry, 35(6), 921-925.

557 Verzele, M. (1986). 100 years of hop chemistry and its relevance to brewing. Journal of the Institute of Brewing, 92(1), 32-48.

559 Wolfe, Peter Harold. (Unpublished results). A study of factors affecting the extraction of flavor when dry hopping beer.

561 
562 Table 1: Variation in the total analysed concentrations of phenolic compounds in 34

563 commercal lager beers.

564

\begin{tabular}{|c|c|c|c|}
\hline \multirow[b]{2}{*}{ BEER } & \multirow[b]{2}{*}{ COUNTRY } & \multicolumn{2}{|c|}{$\mathrm{TPCC}^{\star}(\mathrm{mg} / \mathrm{L})$} \\
\hline & & SUM & SE \\
\hline 0 & USA & 21.17 & 1.5 \\
\hline $\mathbf{Q}$ & USA & 13.12 & 0.8 \\
\hline HH & Czech republic & 18.25 & 1.5 \\
\hline$E$ & Czech republic & 17.73 & 1.8 \\
\hline $\mathbf{L}$ & Czech republic & 13.87 & 1.1 \\
\hline AA & UK & 12.26 & 1.1 \\
\hline $\mathbf{T}$ & USA & 12.73 & 0.6 \\
\hline I & Czech republic & 12.05 & 1.0 \\
\hline w & Belgium & 11.39 & 0.4 \\
\hline $\mathbf{G}$ & Hungary & 11.26 & 0.8 \\
\hline$x$ & Czech republic & 9.61 & 0.2 \\
\hline $\mathbf{M}$ & Italy & 9.17 & 1.0 \\
\hline K & Czech republic & 9.28 & 1.5 \\
\hline $\mathbf{Y}$ & Netherlands & 8.45 & 0.7 \\
\hline $\mathbf{Z}$ & Turkey & 8.18 & 0.4 \\
\hline GG & Romania & 8.89 & 0.5 \\
\hline A & Poland & 8.47 & 0.4 \\
\hline EE & Peru & 8.26 & 0.8 \\
\hline $\mathbf{N}$ & France & 7.44 & 0.7 \\
\hline CC & Italy & 7.30 & 0.5 \\
\hline B & Poland & 7.67 & 0.5 \\
\hline $\mathbf{R}$ & USA & 6.55 & 1.0 \\
\hline DD & Australia & 5.75 & 0.5 \\
\hline $\mathbf{v}$ & UK & 6.45 & 0.5 \\
\hline FF & Cuba & 6.32 & 0.7 \\
\hline $\mathbf{S}$ & Denmark & 5.12 & 0.2 \\
\hline $\mathbf{P}$ & Germany & 6.10 & 0.2 \\
\hline $\mathbf{J}$ & Germany & 5.75 & 0.6 \\
\hline H & Netherlands & 4.79 & 0.5 \\
\hline $\mathbf{U}$ & Netherlands & 5.84 & 1.0 \\
\hline C & South Africa & 5.59 & 0.2 \\
\hline BB & Australia & 4.21 & 0.5 \\
\hline D & South Africa & 4.42 & 0.3 \\
\hline $\mathbf{F}$ & South Africa & 3.91 & 0.1 \\
\hline
\end{tabular}

565

$566 *$ TPCC $=$ total phenolic compound concentration of gallic acid, hydroquinone, protocatechuic acid, catechin, 567 epicatechin, 4-hydroxybenzoic acid, 4-hydroxyphenylacetic acid, caffeic acid, vanillic acid, sinapic acid, syrignic 568 acid, p-coumaric acid, ferulic acid and cinnamic acid quantified in beer by HPLC.

569 SE is standard error of three independent replicate analyses. 
570 Table 2: Beer bitterness descriptors (and their definitions) which were used for sensory

571 evaluation.

\begin{tabular}{llc}
\hline Attribute & Definition & $\boldsymbol{p}$-value \\
\hline Harsh & Tingly, painful, irritating, raspy & $0.000^{*}$ \\
Acidic & Vinegary, fruit-like acidity & 0.491 \\
Tart & Acidic with sour notes & 0.219 \\
Rounded & Pleasant, not spiky, not harsh & $0.000^{*}$ \\
Metallic & Tin/metal taste, silver coin taste & $0.041^{*}$ \\
Sharp & Instant, bitterness taste at tip of tongue & $0.008^{*}$ \\
Smooth & Velvety & $0.006^{*}$ \\
Astringent & Dry, causing drying of the mouth & 0.659 \\
Artificial & Chemically, unnatural beer taste & 0.517 \\
Vegetative & Cabbage, sprout-like bitterness, hop-tea & $0.000^{*}$ \\
Progressive & Bitterness perception increases gradually & $0.00 *^{*}$ \\
Instant & Instantaneous bitterness & $0.020^{*}$ \\
Diminishing & Bitterness perception decreases quickly after ingestion & $0.002^{*}$ \\
\hline
\end{tabular}

$572 p$-values are from Cochran's Q-test. $* P<0.05$ indicates that the term was scored significantly 573 differently amongst the 10 lager beers used for sensory bitterness characterisation. 


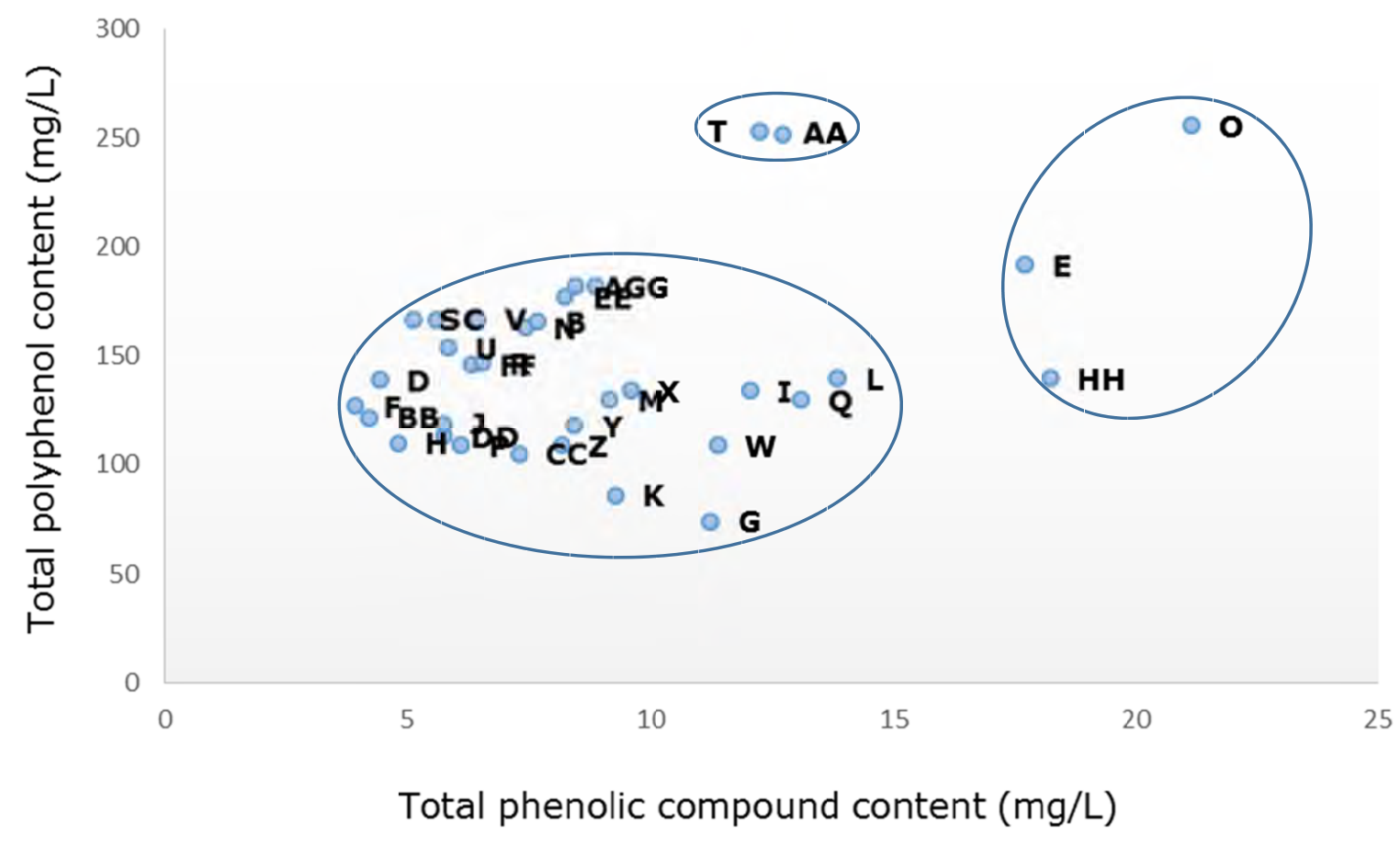

577

581 Figure 1: Plot of total phenolic compound concentrations (HPLC) versus total polyphenol 582 content (according to ASBC method Beer-35) for 34 commercial lager beers. 


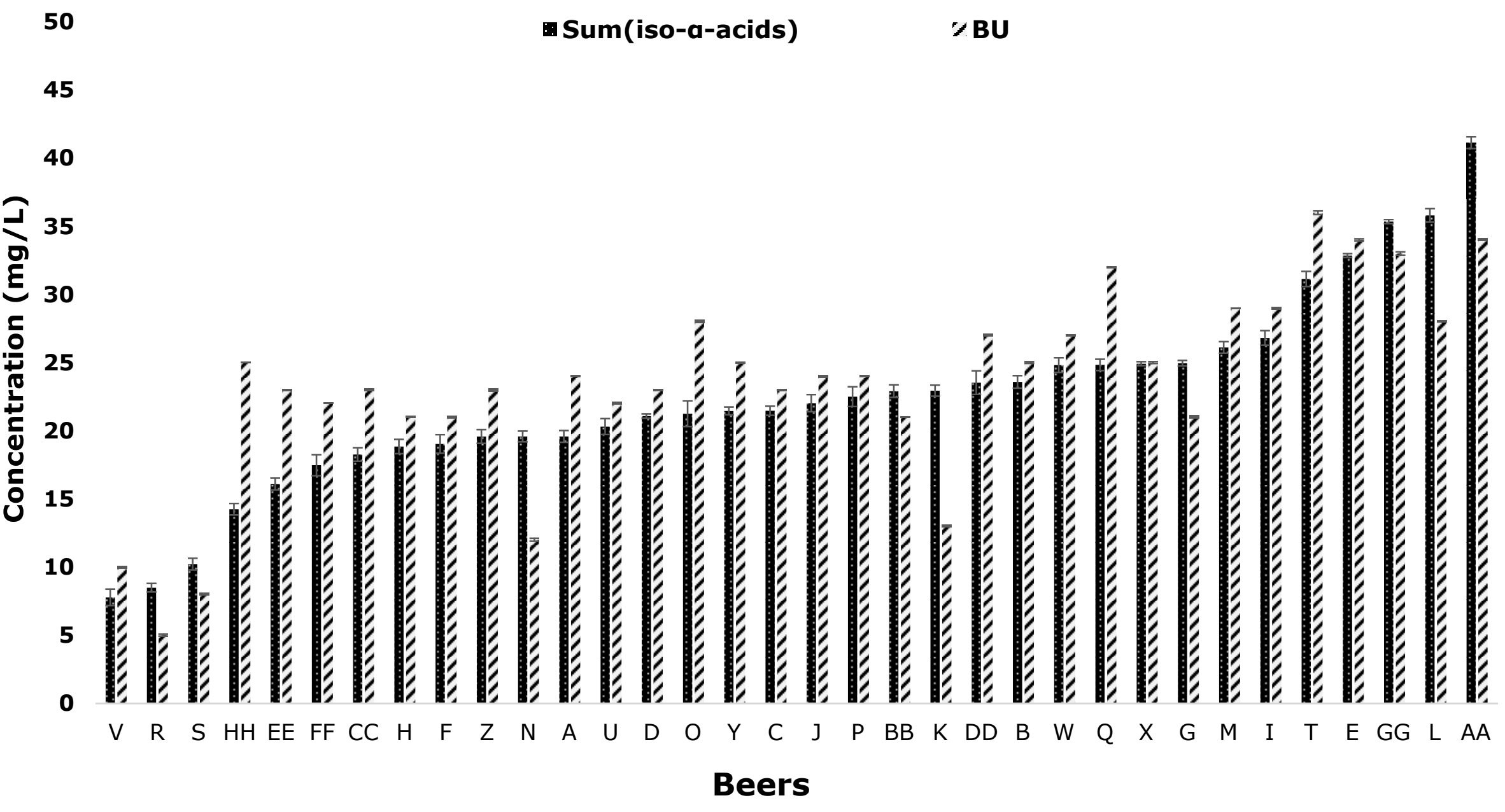

Figure 2: A comparison of the bitterness of 34 commercial lager beers as determined both by spectrophotometric BU values (striped bars) and the sum of iso-humulones determined by HPLC analysis (black bars).

580 Data are ordered by increasing sum of iso- $\alpha$-acids; error bars represent standard error values of 3 independent replicate analyses.

581 *Signifies beers containing tetrahydro-iso- $\alpha$-acids. 


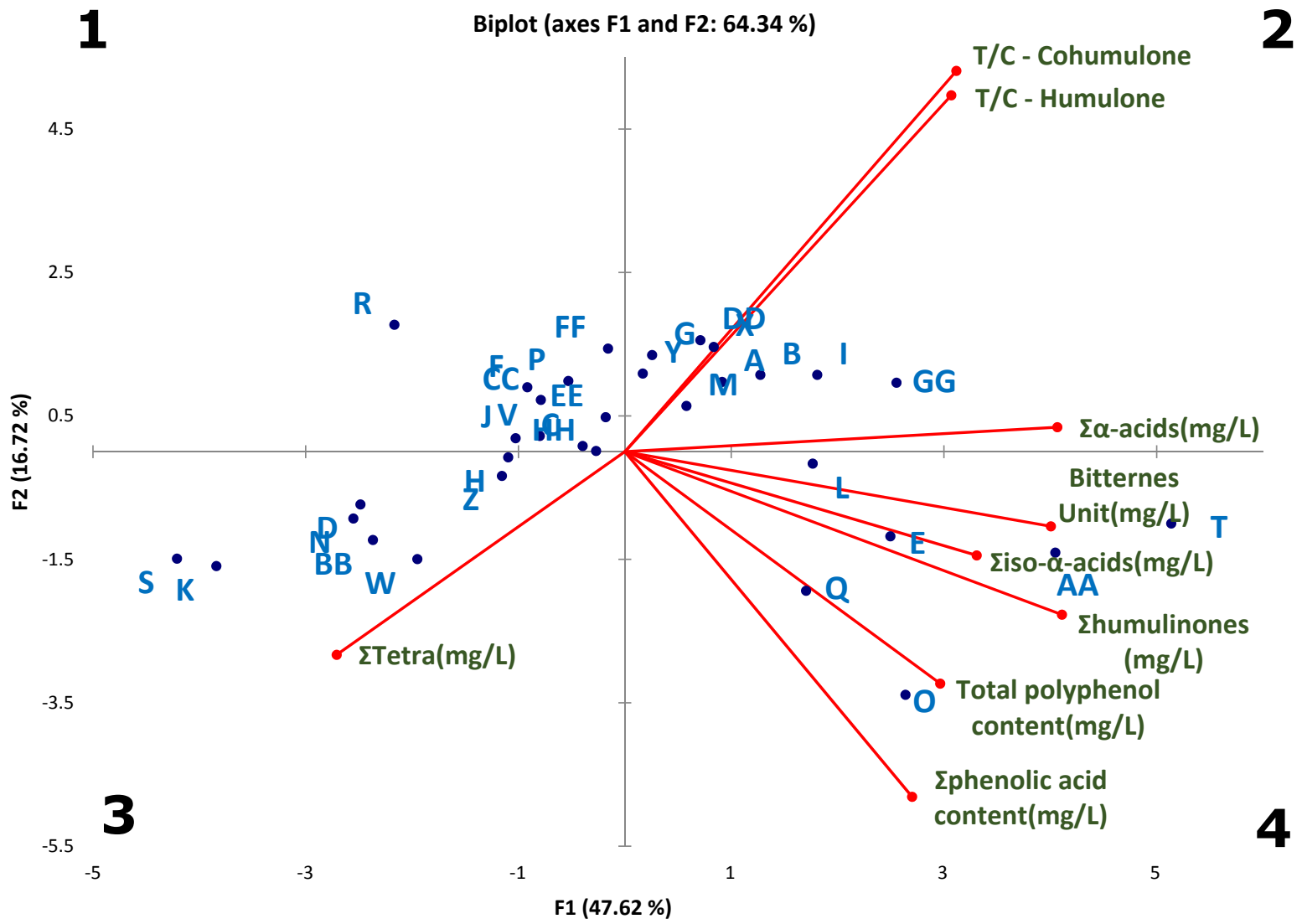

582

\begin{tabular}{c|c|c|c|c|c|c|c|c}
\hline Beer & BU(mg/L) & \multicolumn{3}{|c|}{ Bitterness profile (mg/L) } & TPC(mg/L) & TPCC(mg/L)* & T/C ratio(\%)* \\
& & Humulinones & Iso- $\alpha$-acids & $\boldsymbol{\alpha}$-acids & Tetra & & & \\
\hline CC & 23.0 & 0.0 & 18 & 2.0 & 0.0 & 105.0 & 7.30 & 37 \\
V & 10.0 & 1.5 & 8 & 1.8 & 0.0 & 167.0 & 6.45 & 38 \\
GG & 33.0 & 0.5 & 35 & 3.5 & 0.0 & 182.0 & 8.89 & 47 \\
X & 25.0 & 0.0 & 25 & 1.6 & 0.0 & 134.0 & 9.61 & 48 \\
S & 8.0 & 0.0 & 10 & 0.0 & 3.8 & 167.0 & 5.12 & 27 \\
N & 12.0 & 0.0 & 20 & 0.0 & 3.1 & 163.0 & 7.44 & 34 \\
BB & 21.0 & 0.0 & 23 & 0.0 & 0.0 & 122.0 & 4.21 & 23 \\
E & 34.0 & 0.0 & 33 & 2.2 & 0.0 & 192.0 & 17.73 & 40 \\
AA & 34.0 & 3.0 & 41 & 3.8 & 0.0 & 253.0 & 12.26 & 43 \\
T & 36.0 & 2.5 & 31 & 5.3 & 0.0 & 252.0 & 12.73 & 44 \\
\hline
\end{tabular}

583

584 Figure 3: PCA plot of 34 commercial lager beers according to their analysed contents of

585 hop acid isomers and phenolic compounds. Tabulated data provides a summary of the

586 analytical profile of the beer samples selected for sensory analysis.

$587 *$ TPCC $=$ total phenolic compound concentration of each beer by HPLC. 


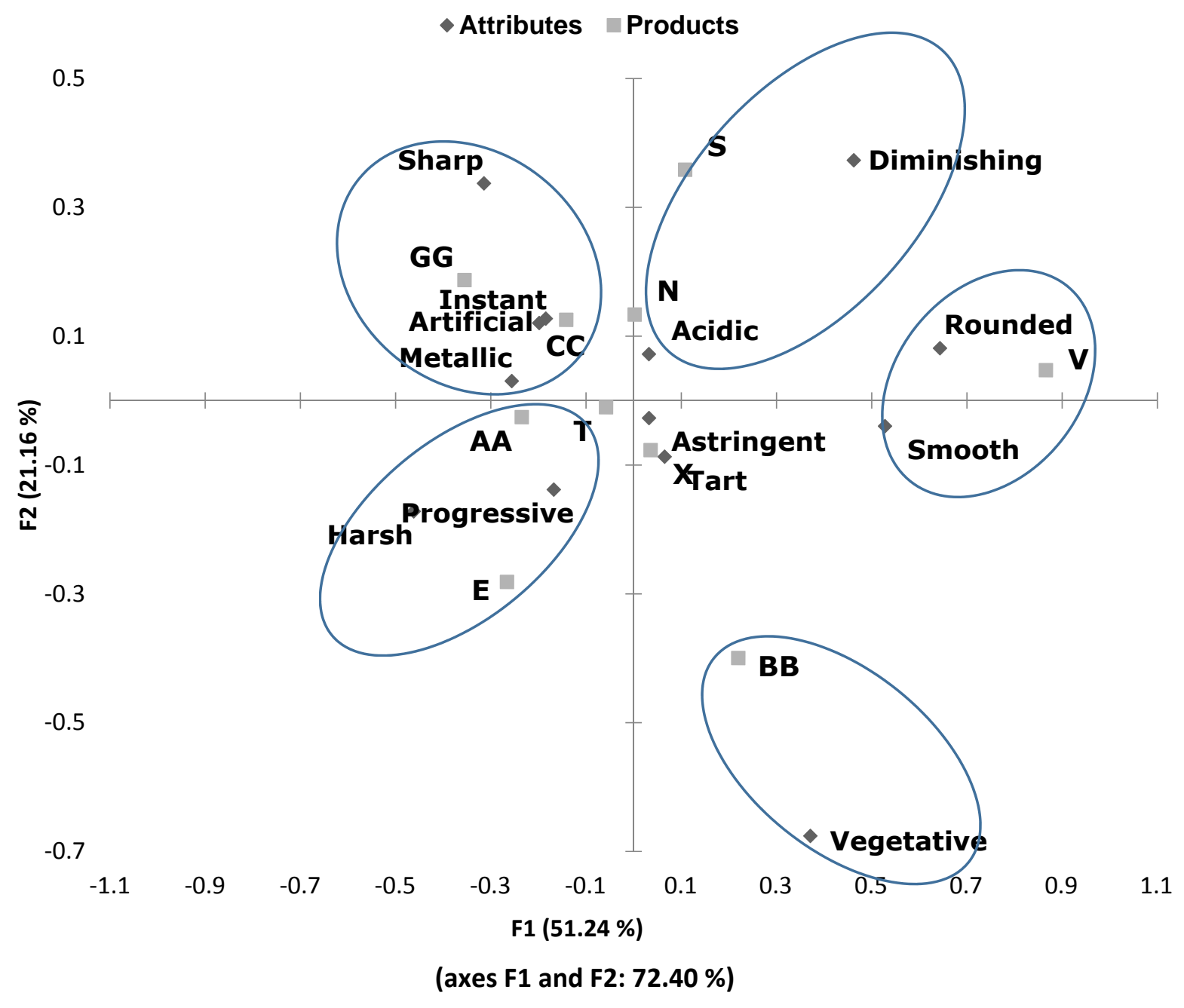

589

590 Figure 4: Correspondence analysis symmetric plot of bitterness attributes (diamonds) 591 and beers (squares). 